\title{
The High-Molecular-Weight Cytochrome $c$ Cyc2 of Acidithiobacillus ferrooxidans Is an Outer Membrane Protein
}

\author{
Andrés Yarzábal, ${ }^{1}$ Gaël Brasseur, ${ }^{2}$ Jeanine Ratouchniak, ${ }^{1}$ Karen Lund, ${ }^{1}$ \\ Danielle Lemesle-Meunier, ${ }^{2}$ John A. DeMoss, ${ }^{1}$ \\ and Violaine Bonnefoy ${ }^{1 *}$ \\ Laboratoire de Chimie Bactérienne ${ }^{1}$ and Laboratoire de Bioénergétique et \\ Ingénierie des Protéines, ${ }^{2}$ CNRS, IBSM, Marseille, France
}

Received 26 June 2001/Accepted 3 October 2001

\begin{abstract}
A high-molecular-weight $c$-type cytochrome, Cyc2, and a putative 22-kDa $c$-type cytochrome were detected in the membrane fraction released during spheroplast formation from Acidithiobacillus ferrooxidans. This fraction was enriched in outer membrane components and devoid of cytoplasmic membrane markers. The genetics, as well as the subcellular localization of $\mathrm{Cyc} 2$ at the outer membrane level, therefore make it a prime candidate for the initial electron acceptor in the respiratory pathway between ferrous iron and oxygen.
\end{abstract}

Acidithiobacillus ferrooxidans, one of the most studied bioleaching bacteria, derives the energy required for its growth mainly from the oxidation of reduced sulfur compounds and/or ferrous iron $\left(\mathrm{Fe}^{2+}\right)(22)$. Although various redox proteins from this organism have been identified and characterized, the electron pathway from $\mathrm{Fe}^{2+}$ to oxygen has not been established. Indeed, several different pathways have been proposed $(3,6$, $17,18,19,23,24,43)$. In each case, the terminal electron acceptor is assumed to be a cytochrome oxidase anchored to the cytoplasmic membrane, and transfer of electrons is postulated to occur through a series of periplasmic carriers, including the copper protein, rusticyanin, and at least one $c$-type cytochrome. However, the nature and the subcellular localization of the primary electron acceptor from $\mathrm{Fe}^{2+}$ still remain in question $(3,6,14,17,18,19,23,24,43)$. In its natural habitat, A. ferrooxidans derives ferrous iron from pyrite, which is insoluble. As previously pointed out $(22,24)$, an excreted and/or an outer membrane (OM) electron transporter can be assumed to be required to link the extracellular pyrite to the periplasmic redox proteins. Based on several arguments, we postulated that the high-molecular-weight cytochrome $c$ encoded by the $c y c 2$ gene (Cyc2) would be located in the OM and could therefore be the first electron carrier in the $\mathrm{Fe}^{2+}$ oxidation respiratory pathway (3). First of all, the $c y c 2$ gene is cotranscribed with the genes encoding an $a a_{3}$-type cytochrome $c$ oxidase, a $21-\mathrm{kDa}$ $c_{4}$-type cytochrome, and rusticyanin, suggesting that these proteins belong to the same pathway (3). Further, the putative signal peptide is removed from Cyc2 in A. ferrooxidans, suggesting its translocation to the periplasm $(2,10)$. Additionally, from the analysis of its amino acid sequence, Cyc2 was predicted to be acid stable and to be located in the OM (3). In support of the postulated role of Cyc2, we present here direct

\footnotetext{
* Corresponding author. Mailing address: Laboratoire de Chimie Bactérienne, CNRS, IBSM, 31 Chemin J. Aiguier, 13402 Marseille Cedex 20, France. Phone: 33 (0)4-91-16-41-46. Fax: 33 (0)4-91-71-8914. E-mail: bonnefoy@ibsm.cnrs-mrs.fr.
}

evidence that the processed holocytochrome is localized in the OM of $A$. ferrooxidans.

Prediction of Cyc2 secondary structure. Cyc2 appears to lack any hydrophobic segments long enough to span the inner membrane (IM) as $\alpha$-helices, based on studies of secondary structure prediction by using multivariate linear regression combination (http://npsa-pbil.ibcp.fr/cgi-bin/secpred mlr.pl) (20), GOR secondary structure prediction (http://molbiol.soton.ac .uk/compute/GOR.html) (15), and prediction of secondary structural content from amino acid composition with analytic vector decomposition (http://www.bork.embl-heidelberg.de/SSCP) (8, 9) algorithms. Rather, this protein is predicted to be composed mainly of $\beta$-sheets, a characteristic of many OM proteins (41).

Preparation and characterization of $\mathrm{OM}$ fractions from $\boldsymbol{A}$. ferrooxidans. To determine the subcellular localization of Cyc2 in $A$. ferrooxidans ATCC 33020, an efficient procedure to resolve membrane fractions was required. Repeated attempts to resolve the crude membranes into the IM and OM fractions by isopyknic sucrose gradients from French press lysates were unsuccessful.

One method, widely used to prepare OM fractions of $A$. ferrooxidans $(7,21,25,32,37,40)$, is the selective disaggregation and solubilization of the IM components by the anionic detergent Sarkosyl (12). However, no conclusive evidence demonstrating the OM nature of Sarkosyl-insoluble fractions or excluding their possible contamination with IM components has been presented to date; generally, the efficiency in OM isolation has been inferred from sodium dodecyl sulfate-polyacrylamide gel electrophoresis (SDS-PAGE) protein profiles. In our studies, the Sarkosyl-insoluble fraction, although featuring some typical properties of an OM fraction, was significantly contaminated with at least one IM protein (cytochrome $c$ oxidase) (data not shown). The failure to resolve efficiently the OM and IM of $A$. ferrooxidans could be due to the composition of its OM, which is particularly rich in lipids (37). We concluded that this procedure could not be used with confidence to localize components specifically to the OM of $A$. ferrooxidans and, therefore, that the localization of proteins 
$\mathbf{A}$

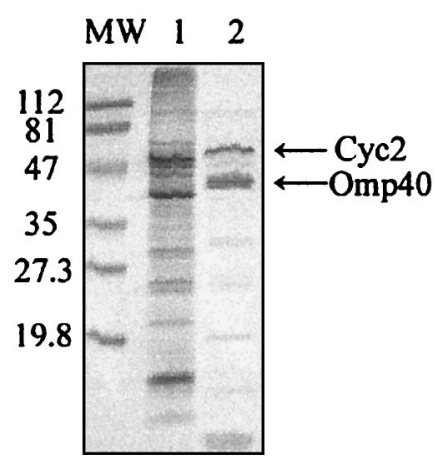

B

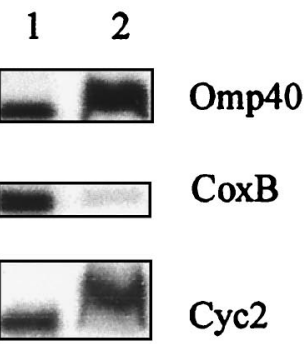

C

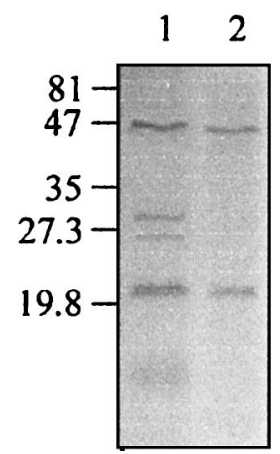

FIG. 1. Characterization of membrane fractions from $A$. ferrooxidans ATCC33020 by SDS-15\% PAGE (27) (A and C) and by Western immunoblotting (B). Total membranes (lanes 1) and OMs (lanes 2) were prepared from $A$. ferrooxidans cells grown in iron medium (3) according to the method of Mizuno and Kageyama (31). In brief, cells were pelleted, washed three times with basal salts, and resuspended in $3 \mathrm{ml}$ of $20 \%$ sucrose at $4^{\circ} \mathrm{C}$. Spheroplasts were obtained by adding sequentially $1.5 \mathrm{ml}$ of $2 \mathrm{M}$ sucrose, $1.7 \mathrm{ml}$ of $0.1 \mathrm{M}$ Tris- $\mathrm{HCl}(\mathrm{pH} 8.0)$, $0.13 \mathrm{ml}$ of $1 \%$ EDTA, $0.15 \mathrm{ml}$ of $10 \mathrm{mg}$ of lysozyme $\mathrm{ml}^{-1}$, and $10 \mu \mathrm{g}$ of DNase $\mathrm{ml}^{-1}$. The mixture was incubated at $30^{\circ} \mathrm{C}$ for $1 \mathrm{~h}$ with occasional shaking. The spheroplasts were removed by centrifugation at $15,000 \times g$ for $15 \mathrm{~min}$; OMs were recovered from the supernatant by centrifugation at $100,000 \times g$ for $90 \mathrm{~min}$ at $4^{\circ} \mathrm{C}$ and then resuspended with distilled water. The remaining spheroplasts were resuspended in $5 \mathrm{mM} \mathrm{MgCl}$ and passed three times through a French press. Total membranes were then recovered by centrifugation at $100,000 \times g$ for $90 \mathrm{~min}$ at $4^{\circ} \mathrm{C}$ after removal of the unbroken cells and debris by lowspeed centrifugation. After SDS-PAGE, gels were stained for total proteins with Coomassie blue (A) or for hemoproteins with $o$-dianisidine (C) (13). The positions of Cyc2 and Omp40 are indicated by arrows in panel A. (B) Western immunoblots were performed with antisera raised against Omp40 (21), purified CoxB, and purified Cyc2 (Bonnefoy et al., unpublished) and visualized with a chemiluminescence kit (ECL Western blotting reagents from Amersham Pharmacia Biotech) based only on the analysis of Sarkosyl-insoluble fractions is questionable.

As an alternative approach, we employed a technique relying on the release into the medium of OM fragments during spheroplast formation (31). This phenomenon seemed likely to occur in A. ferrooxidans, since electron micrographs from a frozen-etched preparation of spheroplasts have shown that the OM was disrupted and partially detached from the cells (1). $A$. ferrooxidans cells were treated with lysozyme and EDTA to produce spheroplasts. After removal of the spheroplasts, the released membranes were pelleted by high-speed centrifugation, resuspended, and analyzed. The SDS-PAGE protein profile of the released membranes displayed a significantly less complex protein band pattern than that of the total membrane (TM) fraction prepared from lysed spheroplasts (Fig. 1A, lanes 1 and 2). Furthermore, while a TM fraction remained at the 40 to $50 \%$ interphase of a discontinuous 30 to $70 \%$ sucrose density gradient, the released membrane fraction migrated to the 60 to $70 \%$ interphase (data not shown), indicating a higher buoyant density as expected for an OM preparation.

Compared to the TM fraction, the released membranes were enriched for specific components of the OM. 2-Keto-3-deoxyoctonate (KDO), a component of the lipopolysaccharides (LPS), quantified by the thiobarbituric acid method (26), was 10 -fold more abundant in the OM fraction $(32.5 \mathrm{mg}$ of $\mathrm{KDO} / \mathrm{g}$ of protein) than in the TM fraction $(3.2 \mathrm{mg}$ of $\mathrm{KDO} / \mathrm{g}$ of protein). These KDO levels are relatively low, as previously reported for $A$. ferrooxidans (44), possibly due to the preparation of membranes at a neutral $\mathrm{pH}$. Indeed, a significant release of LPS has been observed when $A$. ferroxidans cells are incubated at a $\mathrm{pH}$ of $>3.5$ (4). The released membranes were also enriched for Omp40, the major OM porin of $A$. ferrooxidans (21), as shown by Western immunoblotting with a specific antiserum (Fig. 1A and B).

To assess the degree of resolution of the released membrane fraction from IM components, cytochrome $c$ oxidase and $b$ type cytochromes were monitored as IM markers (23). Contrary to what was observed with TM fractions, reduced minus oxidized difference optical spectra of the released OM fraction exhibited no absorption peaks corresponding to $a a_{3}$ cytochrome oxidases (442 and $597 \mathrm{~nm}$ ) or to $b$-type cytochromes (430, 530, and $560 \mathrm{~nm}$ ) (Fig. 2A and B). Furthermore, no detectable cytochrome $c$ oxidase enzymatic activity (28) was detected in the released membrane fraction compared to the TM fraction ( $6 \mu \mathrm{mol}$ of cytochrome $c$ oxidized $\mathrm{min}^{-1} \mathrm{~g}^{-1}$ by using exogenous reduced horse heart cytochrome $c$ as the substrate). Finally, Western immunoblotting with an antiserum raised against the periplasmic domain of the subunit II of the $a a_{3}$ cytochrome oxidase, CoxB, showed only traces of this protein compared to TM levels (Fig. 1B). Based on these results, we conclude that the released membrane fraction represents a well-resolved OM fraction.

Identification and characterization of $\mathrm{OM}$ cytochromes. To detect cytochromes $c$, TM and OM preparations were analyzed

according to the manufacturer's instructions. The retarded migration of the bands in the OM fraction in panels A and B is possibly due to a higher content in LPS. The LMW Electrophoresis Calibration kit from Bio-Rad was used as molecular weight markers (MW). 
$\mathbf{A}$

Room Temp.

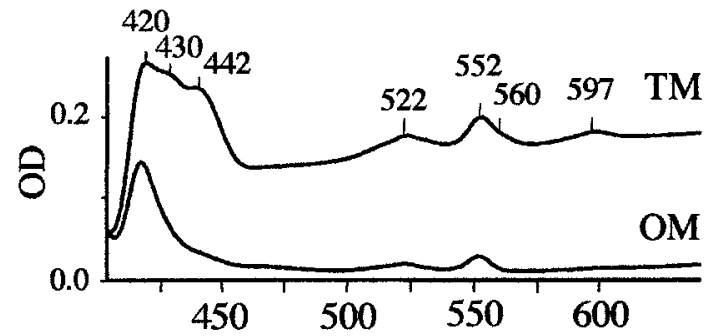

B

Low Temp.

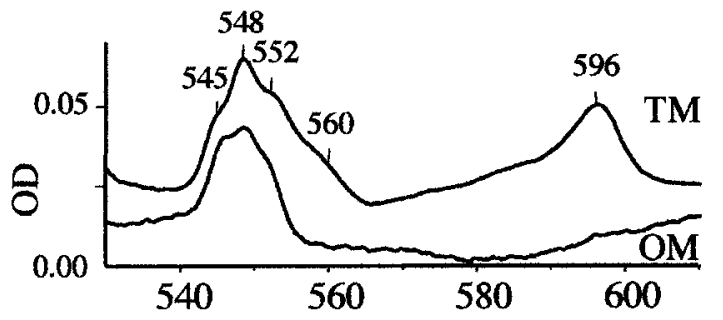

Wavelength (nm)

FIG. 2. Spectrophotometric characterization of membrane fractions of $A$. ferrooxidans. (A) Room temperature optical spectra in the $\alpha, \beta$, and Soret regions. Spectra were obtained as the difference between dithionite-reduced minus $\mathrm{H}_{2} \mathrm{O}_{2}$-oxidized samples. TM and released $\mathrm{OM}$ fractions were suspended at 2.4 and $2.5 \mathrm{mg} \mathrm{ml}^{-1}$, respec-

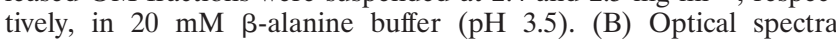
recorded at liquid nitrogen temperature $(77 \mathrm{~K}$ ) in the $\alpha$-band region by using a dual-wavelength DW2000 SLM Aminco spectrophotometer with a slit of $1 \mathrm{~nm}$. Wavelengths were calibrated with a Holmium filter. Spectra were obtained as the difference between dithionite-reduced minus $\mathrm{Na}_{2} \mathrm{IrCl}_{6}$-oxidized samples. TM and $\mathrm{OM}$ fractions were sus-

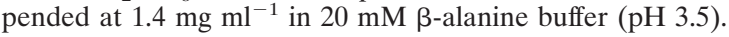

by different procedures. Room temperature spectra showed absorption peaks corresponding to the $\gamma, \beta$, and $\alpha$ bands (420, 522 , and $552 \mathrm{~nm}$ ) of $c$-type cytochromes in both preparations (Fig. 2A). To resolve possible individual components of the $\alpha$ peak, low-temperature $(77 \mathrm{~K})$ difference spectra were recorded (Fig. 2B). Three spectrally distinct $\alpha$ peaks at 545,548 , and 552 $\mathrm{nm}$ (Fig. 2B) were clearly distinguished in the OM. Heme staining with $o$-dianisidine (13) of proteins resolved on SDSPAGE revealed at least five putative $c$-type cytochromes in the TM fraction (Fig. 1C, lane 1), suggesting that these cytochromes are closely associated to the IM and/or the OM. Strikingly, two of these $c$-type cytochromes, the 46- and 22-kDa bands, were associated with the OM fraction (Fig. 1C, lane 2).

The 46-kDa cytochrome $c$ reacted with a Cyc2-specific antiserum (Fig. 1B). Furthermore, microsequencing of the $46-\mathrm{kDa}$ band (473A; Applied Biosystems, Warrington, United Kingdom) yielded an amino-terminal sequence (LPSFARQ) that corresponded to that of the mature Cyc2. The 22-kDa putative $c$-type cytochrome reacted neither with the Cyc2 specific antibodies nor with antibodies directed against the periplasmic $c_{4}$-type cytochrome (data not shown). Because it was not pos- sible to sequence the amino-terminal region, its identity remains unclear. However, the presence of three peaks in the low-temperature spectra (Fig. 2B) suggests that this $22-\mathrm{kDa}$ band corresponds to a dihemic cytochrome because Cyc2 is known to be monohemic (2).

To confirm the localization of Cyc2 in situ, protease digestion of surface proteins with proteinase $\mathrm{K}$ was performed with intact $A$. ferrooxidans cells (11). Cyc2 and Omp40 were significantly degraded after a 2-h incubation (Fig. 3A and B) while no degradation of an IM marker, CoxB, was observed (Fig. 3C). These results not only support the conclusion that Cyc2 is localized in the OM, but they also indicate that some domains of this cytochrome are exposed to the external environment where they may interact with insoluble substrates such as pyrite. Further support for the OM localization of Cyc2 is that, when expressed in Escherichia coli cells, the holocytochrome

\section{Incubation time at $37^{\circ} \mathrm{C}$ (in $\mathrm{min}$ )}

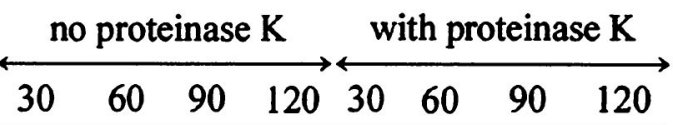

$\mathbf{A}$

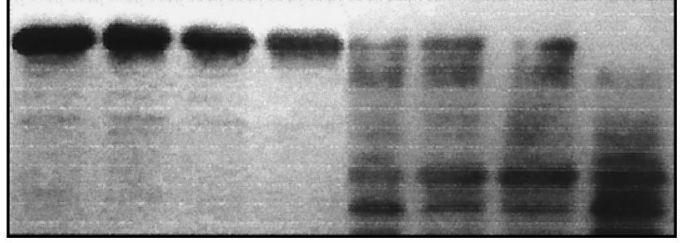

B

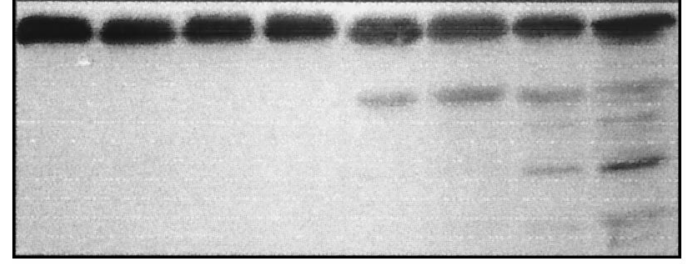

C

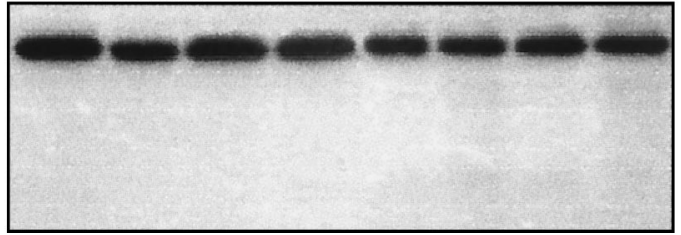

FIG. 3. Localization of Cyc2 in A. ferrooxidans ATCC 33020 cells in situ by proteinase $\mathrm{K}$ digestion of surface proteins (41). Iron-grown bacteria (3) were pelleted and resuspended in phosphate-buffered saline containing $10 \mathrm{mM} \mathrm{MgCl} 2$ at a final concentration of $4 \times 10^{9}$ bacteria $\mathrm{ml}^{-1}$. Proteinase $\mathrm{K}$ was added to a final concentration of 100 $\mu \mathrm{g} \mathrm{ml}^{-1}$, and the mixture was incubated for $30,60,90$, and $120 \mathrm{~min}$ at $37^{\circ} \mathrm{C}$. Digestion was terminated by the addition of $1 \mathrm{mM}$ phenylmethylsulfonyl fluoride plus Laemmli sample buffer (27). The samples were immediately boiled at $100^{\circ} \mathrm{C}$ for $5 \mathrm{~min}$ and then subjected to SDS- $15 \%$ PAGE (27). (A) Protein digestion was assessed by immunodetection on Western blots with anti-Cyc2 polyclonal antiserum (Bonnefoy et al., unpublished) and visualized with a chemiluminescence kit (ECL Western blotting reagents from Amersham Pharmacia Biotech) according to the manufacturer's instructions. Omp40, an OM protein (B), and $\mathrm{CoxB}$, an IM protein (C), were used as positive and negative controls, respectively, and analyzed with the corresponding antisera. Control experiments were performed without any protease added. 
was targeted to the OM (V. Bonnefoy et al., unpublished results).

OM $c$-type cytochrome(s) have been previously described in some neutrophilic microorganisms that also interact with insoluble metals or minerals for energy metabolism, including Desulfovibrio vulgaris (42), Geobacter sulfurreducens (16), Shewanella putrefaciens $(33,34)$, and Shewanella frigidimarina (36). Furthermore, in $S$. putrefaciens, two of these OM $c$-type cytochromes were shown to be required for the activity of the terminal metal reductase $(5,34)$. To our knowledge, this is the first report of OM cytochromes $c$ from an acidophilic microorganism.

In nature, $A$. ferrooxidans must extract electrons from pyrite, an insoluble mineral sulfide. It seems unlikely that iron would be oxidized inside the cell because of the rapid autooxidizability of soluble ferrous iron and the highly insoluble nature of ferric oxide products at the $\mathrm{pH}$ of the cytoplasm or of the periplasm ( $\mathrm{pH} 6.5$ and 3, respectively). Therefore, the site of ferrous oxidation has been inferred to be outside the cell (24) and, indeed, ferric oxides produced during cell growth appear to be deposited outside the cell membranes. Ingledew et al. (24) proposed that electrons derived from the oxidation of ferrous iron are conducted through an extracellular polynuclear iron complex, bound to the phospholipid head groups of the $\mathrm{OM}$, to an acceptor in the periplasmic space. Another hypothesis is that an electron transfer protein would be responsible for the direct oxidation of ferrous iron to ferric iron. Several candidates have been postulated $(3,6,14,17,18,19,23,24$, 43 ), but all of them were periplasmic or bound to the IM, except for Cyc2, which is shown here to be localized in the OM. Therefore, the pathway of electron transfer from ferrous iron to oxygen to drive ATP synthesis is likely Cyc2 rusticyanin $\rightarrow$ Cyc1 $\left(c_{4}\right.$-type cytochrome $) \rightarrow a a_{3}$ cytochrome $c$ oxidase, as we had previously proposed (3). An attractive model for this pathway would be an electron wire spanning both the OM and the IM to conduct electrons from pyrite to oxygen, as has been proposed for the Hmc complex of Desulfovibrio vulgaris subsp. vulgaris Hildenborough (38), and the metal reductase complex of the Shewanella genus $(5,36)$. The genes encoding the electron transporters involved in these complexes are cotranscribed and the corresponding proteins are located in different cellular compartments, as in the present case. According to such a model, the proteins encoded by the cyc2cyc1ORF1coxBACDrus operon (that is, Cyc2, Cyc1, ORF1, the $a a_{3}$-type cytochrome oxidase, and rusticyanin) may also form a transmembrane electron wire allowing electron transfer from the OM Cyc2 to the IM cytochrome oxidase. At this stage, however, we cannot exclude the involvement of excreted redox proteins, as suggested for G. sulfurreducens (39), or the participation of exogenous extracellular molecules $(29,30,35)$, which could transport electrons from the insoluble minerals to the OM.

We thank F. Blasco's group from the Laboratoire de Chimie Bactérienne (Marseille, France) and R. Lloubès from the Laboratoire d'Ingénierie des Systèmes Macromoléculaires (Marseille, France) for helpful advice. We gratefully acknowledge Nicolas Guiliani from the Laboratory of Molecular Microbiology and Biotechnology (Santiago, Chile) for antibodies directed against $A$. ferrooxidans Omp40 protein. We are grateful to R. Lebrun from the IBSM Protein Sequencing Unit (Marseille, France) for performing the N-terminal sequence determination.
A.Y. acknowledges the support of a Graduate Scholarship from the Universidad de los Andes (Merida, Venezuela).

\section{REFERENCES}

1. Apel, W. A., P. R. Dugan, and J. H. Tuttle. 1980. Adenosine $5^{\prime}$-triphosphate formation in Thiobacillus ferrooxidans vesicles by $\mathrm{H}^{+}$ion gradients comparable to those of environmental conditions. J. Bacteriol. 142:295-301.

2. Appia-Ayme, C., A. Bengrine, C. Cavazza, M.-T. Giudici-Orticoni, M. Bruschi, M. Chippaux, and V. Bonnefoy. 1998. Characterization and expression of the co-transcribed $c y c 1$ and $c y c 2$ genes encoding the cytochrome $c_{4}\left(c_{552}\right)$ and a high-molecular-mass cytochrome $c$ from Thiobacillus ferrooxidans ATCC33020. FEMS Microbiol. Lett. 167:171-177.

3. Appia-Ayme, C., N. Guiliani, J. Ratouchniak, and V. Bonnefoy. 1999. Characterization of an operon encoding two $c$-type cytochromes, an $a a_{3}$-type cytochrome oxidase, and rusticyanin in Thiobacillus ferrooxidans ATCC 33020. Appl. Environ. Microbiol. 65:4781-4787.

4. Arredondo, R., A. Garcia, and C. A. Jerez. 1994. Partial removal of lipopolysaccharide from Thiobacillus ferrooxidans affects its adhesion to solids. Appl. Environ. Microbiol. 60:2846-2851.

5. Beliaev, A. S., D. Saffarini, J. McLaughlin, and D. Hunnicutt. 2001. MtrC, an outer membrane decahaem $c$-cytochrome required for metal reduction in Shewanella putrefaciens MR-1. Mol. Microbiol. 39:722-730.

6. Blake, R. C., II, and E. A. Shute. 1994. Respiratory enzymes of Thiobacillus ferrooxidans: kinetic properties of an acid-stable iron:rusticyanin oxidoreductase. Biochemistry 33:9220-9228.

7. Buonfiglio, V., M. Polidoro, L. Flora, G. Citro, P. Valenti, and N. Orsi. 1993. Identification of two outer membrane proteins involved in the oxidation of sulphur compounds in Thiobacillus ferrooxidans. FEMS Microbiol. Rev. 11: 43-50.

8. Eisenhaber, F., F. Imperiale, P. Argos, and C. Froemmel. 1996. Prediction of secondary structural content of proteins from their amino acid composition alone. I. New analytic vector decomposition methods. Proteins Struct. Funct. Design 25:157-168.

9. Eisenhaber, F., C. Froemmel, and P. Argos. 1996. Prediction of secondary structural content of proteins from their amino acid composition alone. II. The paradox with secondary structural class. Proteins Struct. Funct. Design 25:169-179.

10. Elbehti, A., and D. Lemesle-Meunier. 1996. Identification of membranebound $c$-type cytochromes in an acidophilic ferrous ion oxidizing bacterium Thiobacillus ferrooxidans. FEMS Microbiol. Lett. 136:51-56.

11. El-Hage, N., K. Babb, J. A. Carroll, N. Lindstrom, E. R. Fischer, J. C. Miller, R. D. Gilmore, Jr., M. L. Mbow, and B. Stevenson. 2001. Surface exposure and protease insensitivity of Borrelia burgdorferi Erp (OspEF-related) lipoproteins. Microbiology 147:821-830.

12. Filip, C., G. Fletcher, J. L. Wulff, and C. F. Earhart. 1973. Solubilization of the cytoplasmic membrane of Escherichia coli by the ionic detergent sodiumlauryl sarcosinate. J. Bacteriol. 115:717-722.

13. Francis, R. T., Jr., and R. R. Becker. 1984. Specific indication of hemoproteins in polyacrylamide gels using a double-staining process. Anal. Biochem. 136:509-514.

14. Fry, I. V., N. Lazaroff, and L. Packer. 1986. Sulfate-dependent iron oxidation by Thiobacillus ferrooxidans: characterization of a new EPR detectable electron transport component on the reducing side of rusticyanin. Arch. Biochem. Biophys. 246:650-654.

15. Garnier, J., D. J. Osguthorpe, and B. Robson. 1978. Analysis of the accuracy and implications of simple methods for predicting the secondary structure of globular proteins. J. Mol. Biol. 120:97-120.

16. Gaspard, S., F. Vazquez, and C. Holliger. 1998. Localization and solubilization of the iron(III) reductase of Geobacter sulfurreducens. Appl. Environ. Microbiol. 64:3188-3194.

17. Giudici-Orticoni, M.-T., F. Guerlesquin, M. Bruschi, and W. Nitschke. 1999. Interaction-induced redox switch in the electron transfer complex rusticyanin-cytochrome $c_{4}$. J. Biol. Chem. 274:30365-30369.

18. Giudici-Orticoni, M.-T., G. Leroy, W. Nitschke, and M. Bruschi. 2000. Characterization of a new dihemic $c_{4}$-type cytochrome isolated from Thiobacillus ferrooxidans. Biochemistry 39:7205-7211.

19. Giudici-Orticoni, M.-T., W. Nitschke, C. Cavazza, and M. Bruschi. 1997. Characterization and functional role of a cytochrome $c_{4}$ involved in the iron respiratory electron transport chain of Thiobacillus ferrooxidans, p. 4.1-4.10. In Proceedings of the International Biohydrometallurgy Symposium IBS 97/Biomine 97. Australian Mineral Foundation, Glenside, Australia.

20. Guermeur, Y., C. Geourjon, P. Gallinari, and G. Deleage. 1999. Improved performance in protein secondary structure prediction in homogeneous score combination. Bioinformatics 15:413-421.

21. Guiliani, N., and C. A. Jerez. 2000. Molecular cloning, sequencing, and expression of omp-40, the gene coding for the major outer membrane protein from the acidophilic bacterium Thiobacillus ferrooxidans. Appl. Environ. Microbiol. 66:2318-2324.

22. Ingledew, W. J. 1982. Thiobacillus ferrooxidans. The bioenergetics of an acidophilic chemolithotroph. Biochim. Biophys. Acta 683:89-117.

23. Ingledew, W. J., and J. G. Cobley. 1980. A potentiometric and kinetic study 
on the respiratory chain of ferrous-iron-grown Thiobacillus ferrooxidans. Biochim. Biophys. Acta 590:141-158.

24. Ingledew, W. J., J. C. Cox, and P. J. Halling. 1977. A proposed mechanism for energy conservation during $\mathrm{Fe}^{2+}$ oxidation by Thiobacillus ferrooxidans: chemiosmotic coupling to net $\mathrm{H}^{+}$influx. FEMS Microbiol. Lett. 2:193-197.

25. Jerez, C. A., M. Seeger, and A. M. Amaro. 1992. Phosphate starvation affects the synthesis of outer membrane proteins in Thiobacillus ferrooxidans. FEMS Microbiol. Lett. 98:29-34.

26. Karkhanis, Y. D., J. Y. Zeltner, Y. Zeltner, J. J. Jackson, and D. J. Carlo. 1978. A new and improved microassay to determine 2-keto-3-deoxyoctonate in lipopolysaccharide of gram-negative bacteria. Anal. Biochem. 85:595-601.

27. Laemmli, U. K. 1970. Cleavage of structural proteins during the assembly of the head of bacteriophage T4. Nature 227:680-685.

28. Lemesle-Meunier, D., G. Brasseur, P. Tron, D. Bennaroch, W. Nitschke, and A. Elbehti. 2001. The membrane-bound $c$ type cytochromes and the interaction between the downhill and uphill electron transfer pathways in the acidophilic chemolithotrophic ferrous ion-oxidizing bacterium Thiobacillus ferrooxidans, p. 299-308. In V. S. T. Ciminelli and O. Garcia (ed.), Biohydrometallurgy: fundamentals, technology and sustainable development. Proceedings of the International Biohydrometallurgy Symposium, IBS-2001. Elsevier, Amsterdam, The Netherlands.

29. Lovley, D. R., and E. L. Blunt-Harris. 1999. Role of humic-bound iron as an electron transfer agent in dissimilatory Fe(III) reduction. Appl. Environ. Microbiol. 65:4252-4254.

30. Lovley, D. R., J. D. Coates, E. L. Blunt-Harris, E. J. P. Phillips, and J. C. Woodward. 1996. Humic substances as electron acceptors for microbial respiration. Nature 382:445-448.

31. Mizuno, T., and M. Kageyama. 1978. Separation and characterization of the outer membrane of Pseudomonas aeruginosa. J. Biochem. 84:179-191.

32. Mjoli, N., and C. F. Kulpa. 1988. Identification of an unique outer membrane protein required for iron oxidation in Thiobacillus ferrooxidans, p. 89103. In P. R. Norris and D. P. Kelly (ed.), Biohydrometallurgy-Proceedings of the International Symposium Warwick 1987. Science and Technology Letters. Antony Rowe Ltd., Kew, Great Britain.

33. Myers, C. R., and J. M. Myers. 1992. Localization of cytochromes to the outer membrane of anaerobically grown Shewanella putrefaciens MR-1. J. Bacteriol. 174:3429-3438.
34. Myers, C. R., and J. M. Myers. 2001. Role for outer membrane cytochromes OmcA and OmcB of Shewanella putrefaciens MR-1 in reduction of manganese dioxide. Appl. Environ. Microbiol. 67:260-269.

35. Newman, D. K., and R. Kolter. 2000. A role for excreted quinones in extracellular electron transfer. Nature 405:94-97.

36. Richardson, D. J. 2000. Bacterial respiration: a flexible process for a changing environment. Microbiology 146:551-571.

37. Rodriguez, M., S. Campos, and B. Gomez-Silva. 1986. Studies on native strains of Thiobacillus ferrooxidans. III. Studies on the outer membrane of Thiobacillus ferrooxidans. Characterization of the lipopolysaccharide and some proteins. Biotechnol. Appl. Biochem. 8:292-299.

38. Rossi, M., W. B. Pollock, M. W. Reij, R. G. Keon, R. Fu, and G. Voordouw. 1993. The hmc operon of Desulfovibrio vulgaris subsp. vulgaris Hildenborough encodes a potential transmembrane redox protein complex. J. Bacteriol. 175:4699-4711.

39. Seeliger, S., R. Cord-Ruwisch, and B. Schink. 1998. A periplasmic and extracellular $c$-type cytochrome of Geobacter sulfurreducens acts as a ferric iron reductase and as an electron carrier to other acceptors or to partner bacteria. J. Bacteriol. 180:3686-3691.

40. Silva, M., A. Ferreira, M. Rodriguez, and D. Wolf. 1992. The major Thiobacillus ferrooxidans outer membrane protein forms low conductance ion channels in planar lipid bilayers. FEBS Lett. 296:169-173.

41. Van Gelder, P., H. de Cock, and J. Tommassen. 1997. Assembly of bacterial outer membrane proteins, p. 63-82. In G. von Heijne (ed.), Membrane protein assembly. R. G. Landes Co., Austin, Tex.

42. Van Ommen Kloeke, F., R. D. Bryant, and E. J. Laishley. 1995. Localization of cytochromes in the outer membrane of Desulfovibrio vulgaris (Hildenborough) and their role in anaerobic biocorrosion. Anaerobe 1:351-358.

43. Yamanaka, T., Y. Fukumori, T. Yano, M. Kai, and A. Sato. 1991. Enzymatic mechanisms in the "dehydrogenation" of ferrous ions by Thiobacillus ferrooxidans, p. 267-273. In J. Berthelin (ed.), Diversity of environmental biogeochemistry. Elsevier, Amsterdam, The Netherlands.

44. Yokota, A., Y. Yamada, and K. Imai. 1988. Lipopolysaccharides of ironoxidizing Leptospirillum and Thiobacillus ferrooxidans. J. Gen. Appl. Microbiol. 34:27-37. 